\title{
Distributed Averaging with Linear Objective Maps *
}

\author{
Xudong Chen ${ }^{\text {a }}$, M.-A. Belabbas ${ }^{\text {a }}$, Tamer Başar ${ }^{\mathrm{a}}$ \\ ${ }^{a}$ Coordinated Science Laboratory and Electrical and Computer Engineering Department \\ University of Illinois at Urbana-Champaign
}

\begin{abstract}
A distributed averaging system is a linear multi-agent system in which agents communicate to reach an agreement (or a consensus) state, defined as the average of the initial states of the agents. Consider a more generalized situation in which each agent is given a nonnegative weight and the agreement state is defined as the weighted average of the initial conditions. We characterize in this paper the weighted averages that can be evaluated in a decentralized way by agents communicating over a directed graph. Specifically, we introduce a linear function, called the objective map, that defines the desired final state as a function of the initial states of the agents. We then provide a complete answer to the question of whether there is a decentralized consensus dynamics over a given digraph which converges to the final state specified by an objective map. In particular, we characterize not only the set of objective maps that are feasible for a given digraph, but also the consensus dynamics that implements the objective map. In addition, we present a decentralized algorithm to design the consensus dynamics.
\end{abstract}

Key words: Networked Control Systems; Multi-agent Systems; Decentralization.

\section{Introduction}

Distributed averaging has been recognized as an important step in a variety of decentralized and distributed algorithms, such as the rendezvous problem, distributed convex optimization or distributed sensing. We pose and solve in this paper the weighted averaging problem over a directed graph. Specifically, given a set of nonnegative weights assigned to the agents, we say that the agents reach a weighted consensus if they converge to the weighted average of their initial conditions - a formal definition to be given shortly. As is commonly done, we assume that the information flow in the system is described by a directed graph. Our goal is to determine which weighted averages can be computed for a given information flow. Furthermore, we describe how the agents communicate over the graph to design the dynamical system whose evolution reaches the desired agreement state. Computing a weighted average rather than a uniform average is a natural one when the agents in the system

\footnotetext{
* This paper was not presented at any IFAC meeting. Corresponding author: Xudong Chen. T. Başar was partly supported by the U.S. Air Force Office of Scientific Research (AFOSR) MURI grant FA9550-10-1-0573; M.-A. Belabbas was partly supported by NSF ECCS 13-07791 and NSF ECCS CAREER 13-51586; X. Chen was supported jointly by (AFOSR) MURI grant FA955010-1-0573 and by NSF ECCS CAREER 13-51586.

Email addresses: xdchen@illinois.edu (Xudong Chen), belabbas Cillinois.edu (M.-A. Belabbas),

basarleillinois.edu (Tamer Başar).
}

are not all on equal footing. For example, think of a rendezvous problem where the rendezvous position depends on the initial positions of only a small group of agents; of distributed sensing, where the weighting can be proportional to the accuracy of the sensing device, or of opinion dynamics, where participants may have different levels of influence on the decision process. Because of their broad relevance, a fair amount is already known about averaging and consensus algorithms. Indeed, questions concerning sufficient and/or necessary conditions for agents to reach consensus ([1-8]), questions concerning time delay ([3], [8]), consensus with quantized measurements ([9-11]), consensus with time-varying network topologies $([1-8,12])$, and questions about estimating and /or improving convergence rate $([10-15])$ have all been treated to some degree.

Broadly speaking, the problem we address in this paper is one of feasibility of an objective under decentralization constraints. Similar questions, but involving system controllability [16,17], stability of linear systems [18] and formation control $[19,20]$ have also been investigated. While the general problem of feasibility of an objective under decentralization constraints is far from being completely understood, we shall see that a fairly complete characterization can be obtained for the present case, in both discrete- and continuous-time dynamics. However, still open questions remain, such as: How to handle negative weights? How to handle time-varying information flow graphs? How to make sure that no-agent can "game the system" and increase or decrease its assigned weight? 
We next describe the model precisely. We assume that there are $n$ agents $x_{1}, \ldots, x_{n}$ evolving in $\mathbb{R}^{d}$, and that the underlying network topology is specified by a directed graph (or simply digraph) $G=(V, E)$, with $V=\{1, \ldots, n\}$ the set of vertices and $E$ the set of edges. We let $V_{i}^{-}$be a subset of $V$ comprised of the outgoing neighbors of vertex $i$, i.e.,

$$
V_{i}^{-}:=\{j \in V \mid i \rightarrow j \in E\}
$$

and we assume in this paper that each agent $x_{i}$ can only observe its outgoing neighbors. The equations of motion for the $n$ agents $x_{1}, \ldots, x_{n}$ are then given by

$$
\frac{d}{d t} x_{i}=\sum_{j \in V_{i}^{-}} a_{i j}\left(x_{j}-x_{i}\right), \quad \forall i=1, \ldots, n
$$

with each $a_{i j}$ a non-negative real number, which we call the interaction weight.

The objective of the system is characterized by a vector $w=\left(w_{1}, \ldots, w_{n}\right) \in \mathbb{R}^{n}$ of nonnegative entries. We define the linear objective function $f_{w}: \mathbb{R}^{n \times d} \longrightarrow \mathbb{R}^{d}$ as follows:

$$
f_{w}\left(x_{1}, \ldots, x_{n}\right):=\sum_{i=1}^{n} w_{i} x_{i}
$$

The feasibility question we ask is the following: given a digraph $G=(V, E)$, and a weight vector $w=\left(w_{1}, \ldots, w_{n}\right)$ in $\mathbb{R}^{n}$, does there exist a set of non-negative interaction weights $\left\{a_{i j} \mid i \rightarrow j \in E\right\}$ such that for any initial condition $x_{1}(0), \ldots, x_{n}(0)$ in $\mathbb{R}^{d}$, all agents will converge to the same point in $\mathbb{R}^{d}$ specified by the objective map, i.e.,

$$
\lim _{t \rightarrow \infty} x_{i}(t)=f_{w}\left(x_{1}(0), \ldots, x_{n}(0)\right)
$$

for all $i=1, \ldots, n$ ? In other words, we require that all the agents not only reach an agreement, but also converge to a specific point which is a weighted sum of the initial positions of the agents. In the following section, we will convert this problem to one of asking whether there exists a sparse, infinitesimally stochastic matrix $A$ with a fixed zero pattern (specified by the digraph) such that $A$ has a simple zero eigenvalue with $w$ the corresponding left eigenvector.

In the paper, we will provide a complete answer to the question of weighted averaging within model (1). In particular, we characterize both the set of objective maps which are feasible by choosing appropriate interaction weights and, reciprocally, the set of interaction weights for a feasible objective map. The results presented here expand on our earlier work [21] by providing an analysis of the discrete-time case, a finer analysis of the set of interaction weights realizing a feasible linear objective map, a decentralized algorithm for implementing a particular choice of such set, and proofs that were left out. The remainder of the paper is organized as follows. In section 2, we introduce definitions and establish results about the feasibility of linear objective maps over a given network topology. In Section 3, we characterize the sets of interaction weights associated with a feasible linear objective map. A decentralized algorithm for implementing a particular set of such interaction weights is then proposed in Section 4. We also illustrate the algorithm with simulations. We provide conclusions in the last section. The paper ends with an Appendix which contains proofs of some technical results.

\section{Feasibility of Linear Objective Maps}

In this section, we introduce the main definitions used in this work, formulate the weighted averaging problem in precise terms, and characterize the feasible linear objective maps over a given network topology.

\subsection{Background and notations}

We consider in this paper only simple directed graphs, that is directed graphs with no self-loops, and with at most one edge between each ordered pair of vertices. We denote by $G=(V, E)$ a directed graph where $V$ is the vertex set and $E$ is the edge set. Denote by $i \rightarrow j$ an edge of $G$, with $i$ and $j$ the start- and end-vertex of the edge, respectively. A vertex $r$ is said to be a root of $G$ if for all $i \in V$, there is a path from $i$ to $r$. We say that $G$ is rooted if it contains a root. Graphs with only one vertex are by convention rooted. We denote by $V_{r} \subset V$ the set of roots of $G$. The digraph $G$ is strongly connected if, for any ordered pair of vertices $(i, j)$, there is a path from $i$ to $j$. In this case, all vertices of $G$ are roots. It is well known that if the digraph $G$ associated with system (1) is rooted, then all agents converge to the same state for all initial conditions. Conversely, if for any initial condition, all agents of system (1) converge to the same state, then the underlying digraph must be rooted (see, for example, [5, 8]). Hence, for the remainder of the paper, we consider only rooted digraphs as the underlying digraphs of system (1).

For a subset $V^{\prime} \subset V$, we call $G^{\prime}$ a subgraph of $G$ induced by $V^{\prime}$ if $G^{\prime}=\left(V^{\prime}, E^{\prime}\right)$ and $E^{\prime}$ contains any edge of $E$ whose start-vertex and end-vertex are in $V^{\prime}$. We have the following definition:

Definition 1 (Relevant subset) Let $G=(V, E)$ be a rooted digraph. A subset $V^{\prime}$ of $V$ is relevant to $G$ (or simply relevant) if it satisfies the two conditions:

a). The set $V^{\prime}$ is contained in the root set $V_{r}$;

b). The subgraph $G^{\prime}$ induced by $V^{\prime}$ is strongly connected.

For $G$ a digraph with $n$ vertices, we can always let $V=$ $\{1,2, \ldots, n\}$. We denote by $\operatorname{Sp}[V]$ the unit simplex contained in $\mathbb{R}^{n}$ with vertices the standard basis $\left\{e_{1}, \ldots, e_{n}\right\}$ of $\mathbb{R}^{n}$. For a susbet $V^{\prime} \subset V$, we define similarly $\operatorname{Sp}\left[V^{\prime}\right]$ as the convex hull of $\left\{e_{i} \mid i \in V^{\prime}\right\}$ :

$$
\operatorname{Sp}\left[V^{\prime}\right]:=\left\{\sum_{i \in V^{\prime}} \alpha_{i} e_{i} \mid \alpha_{i} \geq 0, \sum_{i \in V^{\prime}} \alpha_{i}=1\right\}
$$

We use the notation $\operatorname{Sp}\left(V^{\prime}\right)$ to denote the interior of $\operatorname{Sp}\left[V^{\prime}\right]$, 
which is defined by the same expression as above, but with $\alpha_{i}>0$ for all $i \in V^{\prime}$. If $V^{\prime}$ is comprised of only one vertex, say vertex $i$, we then set $\operatorname{Sp}\left[V^{\prime}\right]=\operatorname{Sp}\left(V^{\prime}\right)=\left\{e_{i}\right\}$. We note here that if $V_{1}$ and $V_{2}$ are two different subsets of $V$, then $\operatorname{Sp}\left(V_{1}\right)$ and $\operatorname{Sp}\left(V_{2}\right)$ are disjoint. We introduce a similar notation to denote a convex cone. Let $C_{i}$, for $i=1, \ldots, l$, be vectors in $\mathbb{R}^{m}$; we denote the convex cone spanned by $C_{i}$ by

$$
\operatorname{Co}\left[C_{1}, \ldots, C_{l}\right]:=\left\{\sum_{i=1}^{l} \alpha_{i} C_{i} \mid \alpha_{i} \geq 0\right\}
$$

We denote its interior by $\operatorname{Co}\left(C_{1}, \ldots, C_{l}\right)$, which is defined by the same expression as above, but with $\alpha_{i}>0$ for all $i=1, \ldots, l$.

We further need some definitions on infinitesimally stochastic matrices (ISMs). First recall that a square matrix $A$ is said to be an infinitesimally stochastic matrix if its off-diagonal entries are non-negative, and each of its rows sums to zero. We further need the following:

Definition 2 (Graph-induced ISMs) Let $G=(V, E)$ be $a$ digraph with $n$ vertices. Let $\mathbb{A}_{G}$ be a set of $n \times n$ ISMs defined as follows: $A=\left(a_{i j}\right) \in \mathbb{A}_{G}$ if for $i \neq j$,

$$
a_{i j}=\left\{\begin{array}{l}
\geq 0 \text { if } i \rightarrow j \in E, \\
=0 \text { otherwise. }
\end{array}\right.
$$

If $G$ is comprised of only one vertex, then let $\mathbb{A}_{G}:=\{0\}$. We call $\mathbb{A}_{G}$ the set of ISMs induced by $G$.

We note here that the negative of a matrix $A \in \mathbb{A}_{G}$ is also known as the Laplacian matrix of a weighted graph $G$, with the weights being $a_{i j}$ for $i \rightarrow j \in E$. Let 1 be a vector of all ones in $\mathbb{R}^{n}$; then for each matrix $A$ in $\mathbb{A}_{G}$, we have $A \mathbf{1}=0$. So, $A$ has at least one zero eigenvalue. It is also known that if $A$ is an infinitesimally stochastic matrix, then the real parts of eigenvalues of $A$ are non-positive. Given a digraph $G$, we have associated a set of matrices $\mathbb{A}_{G}$. Conversely, given an infinitesimally stochastic matrix, we can define a digraph as follows:

Definition 3 (Matrix-induced graphs) Let $A$ be an $n \times$ $n$ infinitesimally stochastic matrix. A digraph $G=(V, E)$ of $n$ vertices is induced by $A$ if it satisfies the following condition: $i \rightarrow j$ is an edge of $G$ if and only if $a_{i j}$, the $i j-t h$ entry of $A$, is positive.

We further note that an infinitesimally stochastic matrix $A$ has a simple zero eigenvalue if and only if the digraph induced by $A$ is rooted (see, for example, [5]).

\subsection{Main results}

We characterize in this subsection the set of feasible linear objectives maps associated with a digraph $G$. We start by formulating the weighted averaging problem in view of the facts introduced in Subsection 2.1. First, note that we can rewrite (1) into a matrix form as follows: let $X$ be an $n \times$ $d$ matrix with $x_{i}^{\top}$ the $i$-th row of $X$; then, system (1) is equivalent to

$$
\dot{X}=A X
$$

with $A \in \mathbb{A}_{G}$. For the purpose of reaching an agreement, we require that the matrix $A$ have a simple zero eigenvalue. Let $w \in \operatorname{Sp}[V]$ be the left eigenvector of $A$ corresponding to the zero eigenvalue. Then, for any initial condition $X(0)$, we have $\lim _{t \rightarrow \infty} X(t)=\mathbf{1} w^{\top} X(0)$. So, if we write $w=$ $\left(w_{1}, \ldots, w_{n}\right)$, then

$$
\lim _{t \rightarrow \infty} x_{i}(t)=\sum_{i=1}^{n} w_{i} x_{i}(0), \quad \forall i=1, \ldots, n .
$$

Conversely, if the expression above holds for all initial conditions, then the matrix $A$ must have zero as a simple eigenvalue, with $w$ the corresponding left eigenvector. We thus introduce the following subset of $\mathbb{A}_{G}$ :

Definition 4 ( $w$-feasible dynamics) Let $G$ be a rooted digraph, and $w \in \operatorname{Sp}[V]$. The set of $w$-feasible dynamics $\mathbb{A}_{G}(w) \subset \mathbb{A}_{G}$ is defined as follows: if $A \in \mathbb{A}_{G}(w)$, then

1. A has a simple zero eigenvalue.

2. $A^{\top} w=0$.

So the question we raised in the first section can be restated as follows:

Problem 1 For a given digraph $G$ and a vector $w \in \operatorname{Sp}[V]$, is the set $\mathbb{A}_{G}(w)$ empty?

We answer this question in Theorem 1:

Theorem 1 Let $G=(V, E)$ be a rooted digraph, and $V_{1}, \ldots, V_{q}$ be the relevant subsets of $G$. Let $W$ be a subset of $\operatorname{Sp}[V]$ comprised of vectors $w$ for which $\mathbb{A}_{G}(w)$ is nonempty. Then, $W=\bigsqcup_{i=1}^{q} \operatorname{Sp}\left(V_{i}\right)$.

We now consider briefly the case of discrete-time consensus dynamics and show that essentially, the same result holds. To be precise, we consider the model:

$$
X(k+1)=\bar{A} X(k)
$$

where $\bar{A}$ is a stochastic matrix (i.e. its entries are all nonnegative and the sum of the entries in any given row is one). Of course, the matrix $\bar{A}$ is also a sparse matrix, with the zero pattern specified by the rooted graph $\bar{G}=(V, E)$. For simplicity, we assume that $\bar{G}$ has self-loops, i.e., $i \rightarrow i \in E$ for all $i \in V$. Thus, each diagonal entry of $\bar{A}$ is allowed to take a positive value. Similarly, we let $\overline{\mathbb{A}}_{\bar{G}}$ be the collection of the stochastic matrices induced by $\bar{G}$, and let $\overline{\mathbb{A}}_{\bar{G}}(w) \subset$ $\overline{\mathbb{A}}_{\bar{G}}$ be such that each $\bar{A} \in \overline{\mathbb{A}}_{\bar{G}}(w)$ has a simple eigenvalue 1 with $\bar{A}^{\top} w=w$ (note that if $\lambda$ is another eigenvalue of $\bar{A}$, then $|\lambda|<1$ by the Perron-Frobenius theorem). We state following fact as a corollary to Theorem 1 :

Corollary 1 Let $\bar{G}=(V, E)$ be a rooted digraph with a self-loop at each vertex. Let $V_{1}, \ldots, V_{q}$ be the relevant subsets of $\bar{G}$. Let $\bar{W}$ be a subset of $\mathrm{Sp}[V]$ comprised of $w$ for which $\overline{\mathbb{A}}_{\bar{G}}(w)$ is nonempty. Then, $\bar{W}=\bigsqcup_{i=1}^{q} \operatorname{Sp}\left(V_{i}\right)$. 
Proof. Let $G$ be the digraph $\bar{G}$ with self-arcs removed, and let $W$ be the set of $w$ for which $\mathbb{A}_{G}(w)$ is non-empty. We prove Corollary 1 by showing that $\bar{W}=W$ and then appealing to Theorem 1 . We first pick $w \in W$ and let $A \in \mathbb{A}_{G}(w)$. For sufficiently small $\epsilon>0$, we have $(I+\epsilon A) \in \overline{\mathbb{A}}_{\bar{G}}(w)$, and hence $W \subseteq \bar{W}$. Conversely, let $w \in \bar{W}$ and $\bar{A} \in \overline{\mathbb{A}}_{\bar{G}}(w)$; then, $(\bar{A}-I) \in \mathbb{A}_{G}(w)$, and hence $\bar{W} \subseteq W$.

The remainder of the section is devoted to the proof of Theorem 1. We need to show that the following condition:

$$
w \in \bigsqcup_{i=1}^{q} \operatorname{Sp}\left(V_{i}\right)
$$

is both necessary and sufficient for the set $\mathbb{A}_{G}(w)$ to be nonempty. The proof is thus divided into two parts: we establish the necessity and sufficiency of (3) in Subsections 2.3 and 2.4 , respectively.

\subsection{Proof of necessity}

We prove in this subsection that condition (3) is necessary for $\mathbb{A}_{G}(w)$ to be nonempty. To this end, we set

$$
V_{w}:=\left\{i \in V \mid w_{i} \neq 0\right\} .
$$

We establish the following result:

Proposition 1 Let $G=(V, E)$ be a rooted digraph, and $w$ be in $\mathrm{Sp}[V]$. If $\mathbb{A}_{G}(w)$ is nonempty, then $V_{w}$ is relevant to $G$.

The proof of Proposition 1 proceeds by first showing that the subset $V_{w}$ is contained in the root set $V_{r}$ of $G$, and then showing that the subgraph $G_{w}$ induced by $V_{w}$, is strongly connected. This is done in Lemmas 2 and 3 below.

Lemma 2 The subset $V_{w}$ is contained in $V_{r}$.

Proof. Without loss of generality, we may assume that the root set $V_{r}$ consists of the first $m$ vertices. Then, each matrix $A$ in $\mathbb{A}_{G}$ is a lower block-triangular matrix, i.e.,

$$
A=\left(\begin{array}{cc}
A_{11} & 0 \\
A_{21} & A_{22}
\end{array}\right)
$$

with $A_{11}$ an $m \times m$ square matrix and $A_{12}=0$. Indeed, if $a_{i j} \neq 0$ for $1 \leq i \leq m$, then $i \rightarrow j$ is an edge of $G$, and since $i$ is a root, then so is $j$.

In view of the above, the exponential $\exp (A t)$, as the state transition matrix of system (2), is also a lower blocktriangular matrix with blocks of the same dimensions as the blocks of $A$. Furthermore, since the matrix $A$ has a simple zero eigenvalue while all of its other eigenvalues have negative real parts, we have $\lim _{t \rightarrow \infty} \exp (A t)=\mathbf{1} w^{\top}$. Combining this fact with (5), we conclude that $w_{i}=0$ for all $i=m+1, \ldots, n$, and hence $V_{w} \subseteq V_{r}$.
We now proceed to prove the following result:

Lemma 3 The subgraph $G_{w}$ is strongly connected.

Proof. As in the proof of the previous lemma, we may assume without loss of generality that the set $V_{w}$ consists of the first $m$ vertices of $G$. Let $A$ be a matrix in $\mathbb{A}_{G}(w)$, and partition $A$ into $2 \times 2$ blocks as $A=\left(A_{11}, A_{12} ; A_{21}, A_{22}\right)$, with $A_{11}$ being an $m \times m$ matrix and correspondingly, partition $w$ into $w=\left(w^{\prime}, 0\right)$, with $w^{\prime}$ a vector in $\mathbb{R}^{m}$. By assumption, each entry of $w^{\prime}$ is nonzero. Since $A$ is in $\mathbb{A}_{G}(w)$, we have $A^{\top} w=0$; in particular, $A_{12}^{\top} w^{\prime}=0$. Because each entry of $A_{12}$ is non-negative and each entry of $w^{\prime}$ is positive, we must have $A_{12}=0$. This then implies that $A_{11}$ is an $m \times m$ infinitesimally stochastic matrix.

Let $G^{\prime}=\left(V^{\prime}, E^{\prime}\right)$, with $V^{\prime}:=\{1, \ldots, m\}$, be a subgraph of $G$ induced by the block matrix $A_{11}$. Since $G^{\prime}$ is a subgraph of $G_{w}$ on the same set of vertices, it suffices to show that $G^{\prime}$ is strongly connected. To do this, note that the digraph $G^{\prime}$ must be rooted because otherwise $A_{11}$, and hence $A$, has at least two zero eigenvalues. Now, suppose that $G^{\prime}$ is not strongly connected, then the root set of $G^{\prime}$, denoted by $V_{r}^{\prime}$, is a proper subset of $V^{\prime}$. On the other hand, if we let $V_{w^{\prime}}^{\prime}$ be the collection of indices of nonzero entries of $w^{\prime}$, then $V_{w^{\prime}}^{\prime}=V^{\prime}$. But if we apply Lemma 2 to the graph $G^{\prime}$, then $V_{w^{\prime}}^{\prime} \subseteq V_{r}^{\prime}$, and hence

$$
V^{\prime}=V_{w^{\prime}}^{\prime} \subseteq V_{r}^{\prime} \subsetneq V^{\prime}
$$

which is a contradiction. Thus, we conclude that $G^{\prime}$ is strongly connected. This completes the proof.

Combining Lemmas 2 and 3, we establish Proposition 1.

\subsection{Proof of sufficiency}

We prove in this subsection that condition (3) is also sufficient for $\mathbb{A}_{G}(w)$ to be nonempty. Recall that $V_{w}$, for $w \in \operatorname{Sp}[V]$, is the set of indices of nonzero entries of $w$. We establish the following result:

Proposition 2 Let $G$ be a rooted digraph, and $w \in \mathrm{Sp}[V]$. Suppose that $V_{w}$ is relevant to $G$; then, $\mathbb{A}_{G}(w)$ is nonempty.

The proof of Proposition 2 relies on the knowledge of the so-called graph balancing problem for the digraph $G$ (referred to as $G$-balancing). We say that a set of coefficients $b_{i j} \geq 0$, for $i \rightarrow j \in E$, form a solution of $G$-balancing if for each vertex $i$ of $G$, we have

$$
\sum_{k \in V_{i}^{+}} b_{k i}=\sum_{j \in V_{i}^{-}} b_{i j}
$$

with $V_{i}^{+}$and $V_{i}^{-}$being the incoming and the outgoing neighbors of vertex $i$, respectively. We call a solution of $G$ balancing positive if the $b_{i j}$ 's are all positive. We state below a well known fact about the existence of a positive solution of $G$-balancing: 
Lemma 4 Let $G$ be a rooted digraph. There exists a positive solution of $G$-balancing if and only if $G$ is strongly connected.

We refer to [22] for a proof of this fact. We describe below how solutions of $G$-balancing relate to $w$-feasible dynamics. First, note that each nonnegative solution of $G$-balancing $\left\{b_{i j} \mid i \rightarrow j \in E\right\}$ gives rise to an infinitesimally stochastic matrix $B$ defined as follows: let $B_{i j}$ be the $i j$-th entry of $B$; then the off-diagonal entries of $B$ are given by

$$
B_{i j}:= \begin{cases}b_{i j} & \text { if } i \rightarrow j \in E, \\ 0 & \text { otherwise }\end{cases}
$$

and the diagonal entries of $B$ are determined by the condition that rows of $B$ sum to zero. We describe here two relevant properties associated with the matrix $B$ : first, from (6), we have that $B^{\top}$ is an infinitesimally stochastic matrix, and hence $B^{\top} \mathbf{1}=0$; second, if $G$ is strongly connected and $\left\{b_{i j} \mid i \rightarrow j \in E\right\}$ is a positive solution of $G$-balancing, then the associated matrix $B$ has a simple zero eigenvalue.

To proceed, we consider a special case where the digraph $G$ is strongly connected and the vector $w$ lies in $\operatorname{Sp}(V)$ (and hence all entries of $w$ are positive). To this end, let $\operatorname{Diag}(w)$ be a diagonal matrix with $w_{i}$ the $i i$-th entry. Note that since $w \in \operatorname{Sp}(V), \operatorname{Diag}(w)$ is invertible. Denote by $\mathbb{B}_{G}$ the set of infinitesimally stochastic matrices associated with the positive solutions of $G$-balancing. We then establish the following result:

Lemma 5 Let $G$ be strongly connected, and $w \in \operatorname{Sp}(V)$. Then, $\mathbb{A}_{G}(w)$ is nonempty; in particular,

$$
\operatorname{Diag}(w)^{-1} \mathbb{B}_{G} \subseteq \mathbb{A}_{G}(w) .
$$

Proof. First, note that for each $B \in \mathbb{B}_{G}$, the matrix $\operatorname{Diag}(w)^{-1} B$ is in $\mathbb{A}_{G}$. Because $B$ has a simple zero eigenvalue, so does $\operatorname{Diag}(w)^{-1} B$. Then, by the fact that $B^{\top} \mathbf{1}=0$, we obtain

$$
\left(\operatorname{Diag}(w)^{-1} B\right)^{\top} w=B^{\top} \operatorname{Diag}(w)^{-1} w=B^{\top} \mathbf{1}=0,
$$

which implies that $\operatorname{Diag}(w)^{-1} B \in \mathbb{A}_{G}(w)$.

Remark 1 Note that in general, the inequality (7) is strict, i.e., $\operatorname{Diag}(w)^{-1} \mathbb{B}_{G}$ is properly contained in $\mathbb{A}_{G}$. A characterization of $\mathbb{A}_{G}$ is provided in Theorem 2, Section 3.

With Lemma 5 at hand, we prove Proposition 2.

Proof of Proposition 2. Without loss of generality, we assume that $V_{w}=\{1, \ldots, m\}$ with $m \leq n$. Since $V_{w}$ is relevant to $G, V_{w}$ is contained in the root set of $G$ and the subgraph $G_{w}$ induced by $V_{w}$ is strongly connected. Let $w^{\prime} \in \mathbb{R}^{m}$ be defined such that $w=\left(w^{\prime}, 0\right)$.

We now prove that $\mathbb{A}_{G}(w)$ is nonempty by constructing a matrix $A$ in it. Partition the matrix $A$ into $2 \times 2$ blocks as follows:

$$
A=\left(\begin{array}{ll}
A_{11} & A_{12} \\
A_{21} & A_{22}
\end{array}\right)
$$

with $A_{11}$ an $m \times m$ matrix. Recall that $\mathbb{A}_{G_{w}}\left(w^{\prime}\right)$ is the set of $w^{\prime}$-feasible dynamics associated with $G_{w}$. Since $G_{w}$ is strongly connected and all entries of $w^{\prime}$ are positive, we know, from Lemma 5, that the set $\mathbb{A}_{G_{w}}\left(w^{\prime}\right)$ is nonempty, and hence we can pick $A_{11}$ in $\mathbb{A}_{G_{w}}\left(w^{\prime}\right)$. Let $A_{12}$ be the zero matrix. Choose $A_{21}$ and $A_{22}$ such that if $i \rightarrow j$ is an edge of $G$ and if $i>m$, then the $i j$-th entry of $A$ is positive.

We now show that the resulting matrix $A$ is contained in $\mathbb{A}_{G}(w)$. First note that by the choice of $A_{11}$, we have $A_{11}^{\top} w^{\prime}=0$, and hence $A^{\top} w=0$. It now suffices to show that $A$ has zero as a simple eigenvalue. Let $G_{A}$ be the digraph induced by the matrix $A$. We will show that $G_{A}$ is rooted with root set $V^{\prime}$. Since $A_{12}=0$ by construction, there is no edge $i \rightarrow j$ with $1 \leq i \leq m$ and $j>m$. Thus, for any vertex $j \notin V^{\prime}$, there is no path in $G_{A}$ from a vertex $i \in V^{\prime}$ to $j$. So then, the root set of $G_{A}$ is a subset of $V^{\prime}$. On the other hand, each vertex in $V^{\prime}$ is a root of $G$, and by construction of $A_{21}$ and $A_{22}$, we know that if $i \rightarrow j$, for $i>m$, is an edge of $G$, then it is also an edge of $G_{A}$. Thus, for any vertex $i \notin V^{\prime}$, there is a path from $i$ to some vertex in $V^{\prime}$. Since the subgraph $G^{\prime}$ is strongly connected, the set $V^{\prime}$ is the root set of $G_{A}$. Hence, $A$ has zero as a simple eigenvalue. This completes the proof.

Combining Propositions 1 and 2, we establish Theorem 1.

Corollary 2 Let $G$ be a rooted graph, and $w$ be such that $V_{w}$ is a relevant subset. Then,

1. The digraph $G_{A}$ induced by any matrix $A \in \mathbb{A}_{G}(w)$ is rooted, and $V_{w}$ is the root set.

2. The set $\mathbb{A}_{G}(w)$ is convex.

Proof. Letting $A \in \mathbb{A}_{G}(w)$, we show that $G_{A}$ is rooted. For simplicity, but without loss of generality, we assume that $w=\left(w^{\prime}, 0\right)$ with $w^{\prime} \in \mathbb{R}^{m}$ containing the nonzero entries of $w$. Partition $A$ into blocks as we did in (8). Then, $A_{12}=0$ because entries of $A_{12}$ are nonnegative, entries of $w^{\prime}$ are positive, and $A_{12}^{\top} w^{\prime}=0$. Thus, the root set of $G_{A}$ is a subset of $V_{w}$. On the other hand, from Lemma 3, we know that the subgraph $G_{w}$ induced by $V_{w}$ is strongly connected. Thus, the root set of $G_{A}$ is $V_{w}$.

Now let $A^{\prime}$ and $A^{\prime \prime}$ be in $\mathbb{A}_{G}(w)$, and let $A:=\alpha^{\prime} A^{\prime}+$ $\alpha^{\prime \prime} A^{\prime \prime}$, with $\alpha^{\prime}$ and $\alpha^{\prime \prime}$ positive. We show that $A \in \mathbb{A}_{G}(w)$. It should be clear that $A^{\top} w=0$. It remains to show that $A$ has zero as a simple eigenvalue. We prove this fact by showing that $G_{A}$ is rooted. This holds because the two induced digraphs $G_{A^{\prime}}$ and $G_{A^{\prime \prime}}$ are rooted. Hence, the digraph $G_{A}$, as a union of $G_{A^{\prime}}$ and $G_{A^{\prime \prime}}$, is also rooted.

\section{The $w$-feasible Dynamics}

In this section, we assume that the vector $w \in \mathrm{Sp}[V]$ is chosen such that the linear objective map $f_{w}$ is feasible, or 
equivalently, $\mathbb{A}_{G}(w)$-the set of $w$-feasible dynamics-is nonempty. Our goal here is to characterize the set $A_{G}(w)$. We mainly focus on the case when $G$ is strongly connected, and $w$ is in $\operatorname{Sp}(V)$. The analysis relies on the knowledge of the collection of cycles of the digraph $G$. In particular, we introduce in Subsection 3.2 the notion of a principal subset of cycles of $G$. Roughly speaking, a principal subset is defined such that by taking the union of the cycles in the set, we obtain a strongly connected digraph. By introducing principal subsets, we derive a canonical decomposition of $\mathbb{A}_{G}(w)$. We state this characterization precisely in Theorem 2 .

\subsection{On cycles of digraphs}

In this sub-section, we assume that $G=(V, E)$ is an arbitrary digraph. Note that if $G$ is not rooted and $w \in$ $\operatorname{Sp}[V]$, then $\mathbb{A}_{G}(w)$ is empty since no matrix in $\mathbb{A}_{G}$ has zero as a simple eigenvalue. We thus relax this condition in the following definition:

Definition 5 (Relaxed $w$-feasible dynamics) Let $G$ be a digraph, and $w \in \operatorname{Sp}[V]$. We define the set of relaxed $w$-feasible dynamics $\widehat{\mathbb{A}}_{G}(w) \subset \mathbb{A}_{G}$ as follows:

$$
\widehat{\mathbb{A}}_{G}(w):=\left\{A \in \mathbb{A}_{G} \mid A^{\top} w=0\right\}
$$

Our goal in this subsection is to characterize $\widehat{\mathbb{A}}_{G}(w)$. The result derived here will be of great use for investigating the set $\mathbb{A}_{G}(w)$.

Remark 2 We note here that there is a similar type of problems which are related to the characterization of the socalled symmetric transportation polytope (see, for example, [23]). Specifically, let $w \in \mathbb{R}^{n}$ be a nonnegative vector; then the symmetric transportation polytope, denoted by $\mathcal{T}(w)$, is a subset of $\mathbb{R}^{n \times n}$ comprised of nonnegative matrices $M$ with

$$
M \mathbf{1}=M^{\top} \mathbf{1}=w .
$$

For example, if $w=1$, then $M$ is a doubly stochastic matrix, and $\mathcal{T}(\mathbf{1})$ is known as the Birkhoff polytope. Note that if we let $w \in \operatorname{Sp}(V)$ and $\bar{A}:=\operatorname{Diag}(w)^{-1} M$, then from (9) we obtain

$$
\bar{A} \mathbf{1}=\mathbf{1} \quad \text { and } \quad \bar{A}^{\top} w=w .
$$

Thus, the set $\operatorname{Diag}(w)^{-1} \mathcal{T}(w)$ is comprised of stochastic matrices with $w$ the left-eigenvector corresponding to eigenvalue one. There has been a considerable amount of effort toward understanding the polytope $\mathcal{T}(w)$, and especially the extreme points of $\mathcal{T}(w)$. For example, in the case when $\mathcal{T}(w)$ is a Birkhoff polytope, the extreme points of $\mathcal{T}(w)$ are the $n$ ! permutation matrices. We further refer to [23-25] for studies of more general cases. However, we note that the results derived for $\mathcal{T}(w)$ can not be used here to characterize the set $\widehat{\mathbb{A}}_{G}(w)$. Indeed, first note that $\widehat{\mathbb{A}}_{G}(w)$ is comprised of infinitesimally stochastic matrices-in particular, $\widehat{\mathbb{A}}_{G}(w)$ is not a convex polytope, but rather a convex cone (as we will see soon). We also note that all the matrices in $\widehat{\mathbb{A}}_{G}(w)$ are sparse, with the positions of zero entries specified by the digraph $G$. Yet, such a sparse structure is not considered in problems related to symmetric transportation polytopes.

We will now show that $\widehat{\mathbb{A}}_{G}(w)$ is a convex cone and exhibit all of its extreme rays. To proceed, we first introduce some definitions and notations. We say that a digraph $G^{\prime}$ is a cycle of $G$ if $G^{\prime}$ is a subgraph of $G$ and is a cycle with at least two vertices. Let the cycles of $G$ be labelled as $G_{1}, \ldots, G_{k}$. Fix a $w$ in $\operatorname{Sp}(V)$; then, for each cycle $G_{i}$ of $G$, define a matrix $C_{i} \in \mathbb{A}_{G}$ as follows: let $C_{i, j k}$ be the $j k$-th entry of $C_{i}$; then, set

$$
C_{i, j k}:= \begin{cases}1 / w_{j} & \text { if } j \rightarrow k \text { is an edge of } G_{i} \\ 0 & \text { otherwise. }\end{cases}
$$

The diagonal entries of $C_{i}$ are set so that rows of $C_{i}$ sum to zero. Note that by definition, $C_{i}^{\top} w=0$, and hence $C_{i} \in$ $\widehat{\mathbb{A}}_{G}(w)$ for all $i=1, \ldots, k$. To see this, let $v_{j}$ be the $j$-th column of $C_{i}$; then either $v_{j}$ is a zero vector or $v_{j}$ contains two nonzero entries. If $v_{j}$ is a zero vector, then $v_{j}^{\top} w=0$, so we focus on the latter case. By definition of $C_{i}$, the $j$-th entry of $v_{j}$ is $-1 / w_{j}$. Let the other nonzero entry of $v_{j}$ be the $k$-th entry; then, its value is given by $1 / w_{k}$. We thus have

$$
v_{j}^{\top} w=w_{j} \cdot\left(-1 / w_{j}\right)+w_{k} \cdot\left(1 / w_{k}\right)=0 .
$$

This equality holds for each column vector of $C_{i}$, and hence $C_{i}^{\top} w=0$.

Recall that $\mathrm{Co}\left[C_{1}, \ldots, C_{k}\right] \subset \mathbb{R}^{n \times n}$ is the convex cone spanned by the matrices $C_{1}, \ldots, C_{k}$. With the definitions and notations above, we establish the following result:

Proposition 3 Let $G$ be a digraph, and $w$ be in $\operatorname{Sp}(V)$. Let $G_{1}, \ldots, G_{k}$ be the cycles of $G$, and $C_{1}, \ldots, C_{k}$ be the associated infinitesimally stochastic matrices. If $k \geq 1$, then

$$
\widehat{\mathbb{A}}_{G}(w)=\operatorname{Co}\left[C_{1}, \cdots, C_{k}\right]
$$

Moreover, each ray $\left\{\alpha C_{i} \mid \alpha \geq 0\right\}$ is an extreme ray of the convex cone.

By convention, if $k=0$, we set $\widehat{\mathbb{A}}_{G}(w)=\{0\}$. We note here that a similar result relating cycles to doubly stochastic matrices can be found in [26]. We prove Proposition 3 by first investigating a special case where $G$ is acyclic, i.e., there is no cycle contained in $G$.

Lemma 6 Let $G$ be an acyclic digraph, and $w$ be a vector in $\operatorname{Sp}(V)$. Then, $\widehat{\mathbb{A}}_{G}(w)=\{0\}$.

Proof. The proof is carried out by induction on the number of vertices of $G$. For the base case where $G$ is comprised of only one vertex, there is nothing to prove. For the inductive step, we assume that the statement of the lemma holds for $n$, We then prove it for $(n+1)$.

Since $G$ is acyclic, there exists at least a vertex, say vertex 1 , with no incoming edge. Let $A \in \widehat{\mathbb{A}}_{G}(w)$, and let $a_{1}$ 
be the first column of $A$. Then $a_{1}$ has at most one nonzero entry, i.e., the first entry of $a_{1}$. Let $a_{11}$ be the first entry of $a_{1}$; then, $a_{1}^{\top} w=w_{1} a_{11}=0$. Since $w_{1}$ is positive by assumption, we then have $a_{11}=0$. This then implies that the first row vector of $A$ is also a zero vector. Hence, we can partition $A$ into $2 \times 2$ blocks as $A=\left(0,0 ; 0, A^{\prime}\right)$, with $A^{\prime}$ an $n \times n$ matrix.

It now suffices to show that $A^{\prime}$ is a zero matrix. Define

$$
w^{\prime}:=\frac{1}{1-w_{1}}\left(w_{2}, \ldots, w_{n+1}\right) \in \mathbb{R}^{n}
$$

Note that $w^{\prime}$ is well defined because $w_{1}<1$. By construction, $w^{\prime}$ is in the interior of the unit simplex in $\mathbb{R}^{n}$, and moreover, $A^{\prime \top} w^{\prime}=0$. Let $V^{\prime}=\{2, \ldots, n+1\}$, and $G^{\prime}$ be the subgraph induced by $V^{\prime}$. Then, $A^{\prime}$ is in $\widehat{\mathbb{A}}_{G^{\prime}}\left(w^{\prime}\right)$ - the set of relaxed $w^{\prime}$-feasible dynamics associated with $G^{\prime}$. Since the subgraph $G^{\prime}$ is acyclic, from the induction hypothesis, $\widehat{\mathbb{A}}_{G^{\prime}}\left(w^{\prime}\right)$ contains only the zero matrix, and hence $A^{\prime}=0$. This completes the proof.

With Lemma 6 at hand, we prove Proposition 3.

Proof of Proposition 3. First, not that $\mathrm{Co}\left[C_{1}, \cdots, C_{k}\right]$ is contained in $\widehat{\mathbb{A}}_{G}(w)$. This holds because each $C_{i}$ is contained in $\widehat{\mathbb{A}}_{G}(w)$ because $C_{i}^{\top} w=0$.

We now show that $\widehat{\mathbb{A}}_{G}(w)$ is contained in $\operatorname{Co}\left[C_{1}, \cdots, C_{k}\right]$. To this end, fix a matrix $A$ in $\widehat{\mathbb{A}}_{G}(w)$. Suppose that there is a cycle $G_{i}=\left(V_{i}, E_{i}\right)$ in $G$ such that $a_{j k}>0$ for all $j \rightarrow k \in E_{i}$ (we show below that if this is not the case, then $A$ is necessarily the zero matrix). First, note that there exists an $\alpha>0$ such that $\left(A-\alpha C_{i}\right) \in \mathbb{A}_{G}$; indeed the matrix $\left(A-\alpha C_{i}\right)$ is in $\widehat{\mathbb{A}}_{G}(w)$ because $\left(A-\alpha C_{i}\right)^{\top} w=0$. Now let

$$
\alpha_{i}:=\min \left\{w_{j} a_{j k} \mid j \in V_{i}, j \rightarrow k \in E_{i}\right\},
$$

and let $A^{\prime}:=A-\alpha_{i} C_{i}$. Then, $A^{\prime} \in \widehat{\mathbb{A}}_{G}(w)$, and moreover, $A^{\prime}$ has more zero entries than does $A$. To see this, it suffices to check the off-diagonal entries of $A^{\prime}$. First note that if the $j k$-th, $j \neq k$, entry of $A^{\prime}$ is positive, then so is the $j k$-th entry of $A$. Thus, $A^{\prime}$ has at least as many zero entries as $A$ does. Now let

$$
j \rightarrow k \in \operatorname{argmin}\left\{w_{j} a_{j k} \mid j \in V_{i}, j \rightarrow k \in E_{i}\right\}
$$

then, the $j k$-th entry of $A^{\prime}$ is zero because

$$
a_{j k}^{\prime}=a_{j k}-\left(w_{j} a_{j k}\right) C_{i, j k}=a_{j k}-\left(w_{j} a_{j k}\right) 1 / w_{j}=0 .
$$

On the other hand, we have $a_{j k}>0$. Thus, $A^{\prime}$ has more zero entries than does $A$. We then say that the matrix $A^{\prime}$ is a reduction of $A$.

Now let $A \rightarrow A^{(1)} \rightarrow A^{(2)} \rightarrow \ldots$ be a chain of reductions. Since $A^{(k)}$ has more zero entries than $A^{(k-1)}$ does, the chain must be finite. Suppose that this chain stops at $\tilde{A}$, i.e., there does not exist a reduction of $\tilde{A}$. It then suffices to prove that $\tilde{A}$ is a zero matrix. Let $\tilde{G}$ be a digraph with $n$ vertices induced by the matrix $\tilde{A}$. Since there is no reduction of $\tilde{A}$, the induced digraph $\tilde{G}$ must be acyclic. Since $\tilde{A}^{\top} w=0$ with $w \in \operatorname{Sp}(V)$, by Lemma 6 , we have $\tilde{A}=0$.

It now remains to show that each ray $\left\{\alpha C_{i} \mid \alpha \geq 0\right\}$ is an extreme ray of the convex cone $\widehat{\mathbb{A}}_{G}(w)$. We show that for each matrix $C_{i}$, there does not exist a set of non-negative coefficients $\alpha_{j}$ 's such that $C_{i}=\sum_{j \neq i} \alpha_{j} C_{j}$. We prove by contradiction. Suppose that the expression above holds; then at least one coefficient $\alpha_{j}$, for $j \neq i$, is positive. Hence, the cycle $G_{j}$ is a subgraph of $G_{i}$. On the other hand, $G_{i}$ itself is a cycle, so we must have $G_{i}=G_{j}$, which is a contradiction. This completes the proof.

\subsection{On principal subsets}

In this section, we assume that $G=(V, E)$ is a strongly connected digraph with $n$ vertices for $n>1$. Let $\mathcal{G}=$ $\left\{G_{1}, \ldots, G_{k}\right\}$ be the set of cycles of $G ; \mathcal{G}$ is non-empty since for each edge $i \rightarrow j$ of $G$, there is at least one cycle containing that edge. Recall that a Hamiltonian cycle of $G$ is a cycle that passes through every vertex. We can thus say that this cycle strongly connects the graph, in the sense that each vertex is connected to every other vertex using only edges in the cycle. If $G$ does not have a Hamiltonian cycle, one can nevertheless use several cycles to strongly connect $G$. We make this formal through the introduction of a principal subset of $\mathcal{G}$, which is defined as follows:

Definition 6 (Principal subsets) Let $G$ be a digraph and $\mathcal{G}=\left\{G_{1}, \ldots, G_{k}\right\}$ be its set of cycles. Denote by $G_{i}=$ $\left(V_{i}, E_{i}\right)$. A subset $\left\{G_{1}^{\prime}, \ldots, G_{m}^{\prime}\right\}$ of $\mathcal{G}$ is principal if the graph $G^{\prime}=\left(V, \cup_{i} E_{i}^{\prime}\right)$ is strongly connected.

For the remainder of the section, we label the principal subsets of $\mathcal{G}$ as $\mathcal{G}_{1}, \ldots, \mathcal{G}_{p}$. Let $\mathcal{G}_{i}=\left\{G_{i_{1}}, \ldots, G_{i_{m}}\right\}$ be a principal subset of $\mathcal{G}$. Fix a vector $w \in \operatorname{Sp}(V)$, and let $C_{i_{1}}, \ldots, C_{i_{m}}$ be the associated infinitesimally stochastic matrices (with entries defined in (10)). Recall that $\mathrm{Co}\left[C_{i_{1}}, \ldots, C_{i_{m}}\right]$ is the convex cone spanned by $C_{i_{1}}, \ldots, C_{i_{m}}$. With a slight abuse of notation, we write

$$
\mathrm{Co}\left[\mathcal{G}_{i}\right]:=\mathrm{Co}\left[C_{i_{1}}, \ldots, C_{i_{m}}\right]
$$

Similarly, we denote the interior of $\operatorname{Co}\left[\mathcal{G}_{i}\right]$ by $\operatorname{Co}\left(\mathcal{G}_{i}\right)$. Equipped with the definitions and notations above, we establish the following result:

Lemma 7 Let $G$ be a strongly connected digraph with $n$ vertices for $n>1$, and let $\mathcal{G}_{1}, \ldots, \mathcal{G}_{p}$ be principal subsets of $\mathcal{G}$. Let $w \in \operatorname{Sp}(V)$. Then, $\mathbb{A}_{G}(w)=\bigcup_{i=1}^{p} \operatorname{Co}\left(\mathcal{G}_{i}\right)$.

Proof. We first show that each set $\operatorname{Co}\left(\mathcal{G}_{i}\right)$ is contained in $\mathbb{A}_{G}(w)$. Suppose that $\mathcal{G}_{i}$ is comprised of cycles $G_{i_{1}}, \ldots, G_{i_{m}}$. For any matrix $A$ in $\operatorname{Co}\left(\mathcal{G}_{i}\right)$, there exists a set of positive coefficients $\alpha_{1}, \ldots, \alpha_{m}$ such that 
$A=\sum_{j=1}^{m} \alpha_{j} C_{i_{j}}$. Let $G_{A}$ be the digraph induced by matrix $A$; then $G_{A}$ is strongly connected by the definition of principal subset. Consequently, the matrix $A$ has a simple zero eigenvalue. Furthermore, by the definition of $C_{i_{j}}$, we have that $C_{i_{j}}^{\top} w=0$ for all $j=1, \ldots, m$. So, $A^{\top} w=0$, and hence $A \in \mathbb{A}_{G}(w)$.

Next we show that the set $\mathbb{A}_{G}(w)$ is contained in the union of $\operatorname{Co}\left(\mathcal{G}_{1}\right), \ldots, \operatorname{Co}\left(\mathcal{G}_{p}\right)$. Let $A$ be a matrix in $\mathbb{A}_{G}(w)$; then $A$ is also contained in $\widehat{\mathbb{A}}_{G}(w)$. Thus by Proposition 3 , there is a set of non-negative coefficients $\alpha_{1}, \ldots, \alpha_{k}$ such that $A=\sum_{i=1}^{k} \alpha_{i} C_{i}$. Suppose $\alpha_{i_{1}}, \ldots, \alpha_{i_{m}}$ are the non-zero coefficients out of $\alpha_{1}, \ldots, \alpha_{k}$; then, let $\mathcal{G}^{\prime}:=\left\{G_{i_{1}}, \ldots, G_{i_{m}}\right\}$. We need to show that $\mathcal{G}^{\prime}$ is a principal subset of $\mathcal{G}$. Let $G_{A}$ be the digraph induced by $A$; then, $G_{A}$ is the union of $G_{i_{1}}, \ldots, G_{i_{m}}$. It now suffices to show that $G_{A}$ is strongly connected. Suppose that it is not the case; then, by Proposition 1 , the set $\mathbb{A}_{G}(w)$ is empty because $w \in \operatorname{Sp}(V)$, and hence $V_{w}=V$ which is not relevant to $G_{A}$. On the other hand, $A \in \mathbb{A}_{G}(w)$, which is a contradiction. Hence, $G_{A}$ is strongly connected, and thus $\mathcal{G}^{\prime}$ is a principal subset of $\mathcal{G}$.

We note that in general, the right-hand-side of the decomposition of $\mathbb{A}_{G}(w)$ given in Lemma 7 may contain redundant terms. Indeed, we might have for some $i$ such that

$$
\mathrm{Co}\left(\mathcal{G}_{i}\right) \subseteq \cup_{j \neq i} \operatorname{Co}\left(\mathcal{G}_{j}\right) .
$$

To rule out this redundancy, we introduce the following definition:

Definition 7 (Minimal Cover) For a collection of arbitrary sets $\left\{\mathcal{A}_{i}\right\}_{i=1}^{p}$, let $\mathcal{A}=\cup_{i=1}^{p} \mathcal{A}_{i}$. A collection $\left\{\mathcal{A}_{i_{1}}, \ldots, \mathcal{A}_{i_{l}}\right\} \subset\left\{\mathcal{A}_{i}\right\}_{i=1}^{p}$ is a minimal cover of $\mathcal{A}$ if $\mathcal{A}=\cup_{j=1}^{l} \mathcal{A}_{i_{j}}$ and $l$ is the least integer for the relation to hold.

For the collection of sets $\operatorname{Co}\left(\mathcal{G}_{1}\right), \ldots, \operatorname{Co}\left(\mathcal{G}_{p}\right)$, it should be clear that the minimal cover of $\mathbb{A}_{G}(w)$ always exists. We now show that there exists a unique minimal cover of $\mathbb{A}_{G}(w)$ and its components are pairwise disjoint. To do so, we define a partial order over the set of principal subsets as follows: let us introduce the notation

$$
\mathcal{G}_{i} \succ \mathcal{G}_{j} \quad \text { if } \quad \operatorname{Co}\left(\mathcal{G}_{i}\right) \supsetneq \operatorname{Co}\left(\mathcal{G}_{j}\right) .
$$

A principal subset $\mathcal{G}^{\prime}$ is said to be a maximal element with respect to the partial order if there does not exist another principal subset $\mathcal{G}^{\prime \prime}$ such that $\mathcal{G}^{\prime \prime} \succ \mathcal{G}^{\prime}$. We label $\mathcal{G}_{1}^{*}, \ldots, \mathcal{G}_{l}^{*}$ as the maximal elements among all principal subsets. With the definitions above, we establish the following result.

Theorem 2 Let $G=(V, E)$ be a strongly connected digraph of $n$ vertices with $n>1$, and let $w \in \operatorname{Sp}(V)$. Then, the collection $\mathcal{S}:=\left\{\mathrm{Co}\left(\mathcal{G}_{1}^{*}\right), \ldots, \mathrm{Co}\left(\mathcal{G}_{l}^{*}\right)\right\}$ is the unique minimal cover of $\mathbb{A}_{G}(w)$. Furthermore, the sets in $\mathcal{S}$ are pairwise disjoint.

We establish below Theorem 2. We start by proving the following result.
Lemma 8 If $\mathcal{G}_{i} \succ \mathcal{G}_{j}$, then $\mathcal{G}_{i} \supsetneq \mathcal{G}_{j}$.

Proof. It suffices to show that $\mathcal{G}_{i} \supseteq \mathcal{G}_{j}$. Suppose it is not the case, and that there exists a cycle $G_{k}$ such that $G_{k} \in \mathcal{G}_{j}-\mathcal{G}_{i}$. By Proposition 3, $\left\{\alpha C_{k} \mid \alpha \geq 0\right\}$ is an extreme ray of $\widehat{\mathbb{A}}_{G}(w)$. This, in particular, implies that $C_{k} \notin \mathrm{Co}\left[\mathcal{G}_{i}\right]$. On the other hand, if $\mathcal{G}_{i} \succ \mathcal{G}_{j}$, then $\mathrm{Co}\left[\mathcal{G}_{i}\right] \supseteq \operatorname{Co}\left[\mathcal{G}_{j}\right]$. But then,

$$
C_{k} \in \mathrm{Co}\left[\mathcal{G}_{i}\right]-\operatorname{Co}\left[\mathcal{G}_{j}\right]=\varnothing
$$

which is a contradiction. This completes the proof.

The converse of Lemma 8 is not true in general, i.e., we may not conclude that $\mathcal{G}_{i} \succ \mathcal{G}_{j}$ from the condition that $\mathcal{G}_{i} \supsetneq \mathcal{G}_{j}$. However, we will be able to prove that if $\mathcal{G}_{i} \supsetneq \mathcal{G}_{j}$, then either $\mathcal{G}_{i} \succ \mathcal{G}_{j}$ or $\operatorname{Co}\left(\mathcal{G}_{i}\right) \cap \operatorname{Co}\left(\mathcal{G}_{j}\right)=\varnothing$. To proceed, we define for a given principal subset $\mathcal{G}^{\prime}=\left\{G_{i_{1}}, \ldots, G_{i_{m}}\right\}$ a linear subspace of $\mathbb{R}^{n \times n}$ :

$$
L\left(\mathcal{G}^{\prime}\right):=\left\{\sum_{j=1}^{m} \beta_{j} C_{i_{j}} \mid \beta_{j} \in \mathbb{R}\right\} .
$$

Note that each $\operatorname{Co}\left(\mathcal{G}_{i}\right)$ is an open subset of $L\left(\mathcal{G}_{i}\right)$. It should be clear that if $\mathcal{G}_{i} \supseteq \mathcal{G}_{j}$, then $L\left(\mathcal{G}_{i}\right) \supseteq L\left(\mathcal{G}_{j}\right)$. Furthermore, we have the following fact:

Lemma 9 Let $\mathcal{G}_{i} \supsetneq \mathcal{G}_{j}$, and $\mathcal{G}^{\prime}:=\mathcal{G}_{i}-\mathcal{G}_{j}$. Then, the following hold:

1. If $L\left(\mathcal{G}_{i}\right)=L\left(\mathcal{G}_{j}\right)$, then $\mathcal{G}_{i} \succ \mathcal{G}_{j}$.

2. If $L\left(\mathcal{G}_{i}\right) \supsetneq L\left(\mathcal{G}_{j}\right)$ and $\operatorname{Co}\left(\mathcal{G}^{\prime}\right) \cap L\left(\mathcal{G}_{j}\right) \neq \varnothing$, then $\mathcal{G}_{i} \succ \mathcal{G}_{j}$.

3. If $L\left(\mathcal{G}_{i}\right) \supsetneq L\left(\mathcal{G}_{j}\right)$ and $\operatorname{Co}\left(\mathcal{G}^{\prime}\right) \cap L\left(\mathcal{G}_{j}\right)=\varnothing$, then $\mathrm{Co}\left(\mathcal{G}_{i}\right) \cap \operatorname{Co}\left(\mathcal{G}_{j}\right)=\varnothing$.

We refer to the Appendix for a proof of Lemma 9. With Lemmas 8 and 9 at hand, we prove Theorem 2:

Proof of Theorem 2. First, we show that $\mathcal{S}$ is a cover of $\mathbb{A}_{G}(w)$. Since the $\mathcal{G}_{i}^{*}$ 's are maximal elements, each set $\mathrm{Co}\left(\mathcal{G}_{j}\right)$, for $j=1, \ldots, p$, is contained in some $\operatorname{Co}\left(\mathcal{G}_{i}^{*}\right)$ for $i=1, \ldots, l$. By Lemma $7, \mathbb{A}_{G}(w)=\bigcup_{i=1}^{p} \operatorname{Co}\left(\mathcal{G}_{i}\right)$, which implies that $\mathcal{S}$ is a cover of $\mathbb{A}_{G}(w)$.

Next, we show that the sets $\operatorname{Co}\left(\mathcal{G}_{1}^{*}\right), \ldots, \mathrm{Co}\left(\mathcal{G}_{l}^{*}\right)$ are pairwise disjoint. We prove this by contradiction. Assume that there is a matrix $A \in \operatorname{Co}\left(\mathcal{G}_{i}^{*}\right) \cap \operatorname{Co}\left(\mathcal{G}_{j}^{*}\right)$ for a pair of distinct indices $(i, j)$. Let $\mathcal{G}^{\prime}:=\mathcal{G}_{i}^{*} \cup \mathcal{G}_{j}^{*}$; we show that $A \in \operatorname{Co}\left(\mathcal{G}^{\prime}\right)$. To this end, we let $\mathcal{G}_{i}^{*}=\left\{G_{i_{1}}, \ldots, G_{i_{m}}\right\}$ and $\mathcal{G}_{j}^{*}=\left\{G_{j_{1}}, \ldots, G_{j_{m^{\prime}}}\right\}$. Then, there are positive coefficients $\alpha_{i_{t}}$ 's and $\beta_{j_{s}}$ 's such that $A=\sum_{t=1}^{m} \alpha_{i_{t}} C_{i_{t}}=$ $\sum_{s=1}^{m^{\prime}} \beta_{j_{s}} C_{j_{s}}$. We can thus express $A$ as follows

$$
A=\frac{1}{2} \sum_{t=1}^{m} \alpha_{i_{t}} C_{i_{t}}+\frac{1}{2} \sum_{s=1}^{m^{\prime}} \beta_{j_{s}} C_{j_{s}} .
$$


which shows that $A \in \operatorname{Co}\left(\mathcal{G}^{\prime}\right)$. Since the $\mathcal{G}_{k}^{*}$ 's are distinct, we have $\mathcal{G}^{\prime} \supsetneq \mathcal{G}_{i}^{*}$ and/or $\mathcal{G}^{\prime} \supsetneq \mathcal{G}_{j}^{*}$. We assume without loss of generality that the former holds. By Lemma 9, either $\mathcal{G}^{\prime} \succ \mathcal{G}_{i}^{*}$ or $\operatorname{Co}\left(\mathcal{G}^{\prime}\right) \cap \mathrm{Co}\left(\mathcal{G}_{i}^{*}\right)=\varnothing$. But since

$$
A \in \operatorname{Co}\left(\mathcal{G}^{\prime}\right) \cap \operatorname{Co}\left(\mathcal{G}_{i}^{*}\right),
$$

we conclude that $\mathcal{G}^{\prime} \succ \mathcal{G}_{i}^{*}$, which then contradicts the fact that $\mathcal{G}_{i}^{*}$ is a maximal element. Thus, we have proved that $\mathrm{Co}\left(\mathcal{G}_{1}^{*}\right), \ldots, \mathrm{Co}\left(\mathcal{G}_{l}^{*}\right)$ are pairwise disjoint.

It now remains to show that $\mathcal{S}$ is a minimal cover, and indeed it is the unique minimal cover. Let $\left\{\mathcal{G}_{1}^{\prime}, \ldots, \mathcal{G}_{l^{\prime}}^{\prime}\right\}$ be any set of principal subsets such that

$$
\left\{\mathrm{Co}\left(\mathcal{G}_{1}^{\prime}\right), \ldots, \operatorname{Co}\left(\mathcal{G}_{l^{\prime}}^{\prime}\right)\right\}
$$

is a minimal cover of $\mathbb{A}_{G}(w)$. Then, we have $l^{\prime} \leq l$. Now, to each $\mathcal{G}_{i}^{\prime}$, we can assign a maximal element $\overline{\mathcal{G}_{i}^{\prime}} \in\left\{\mathcal{G}_{1}^{*}, \ldots, \mathcal{G}_{l}^{*}\right\}$ such that $\overline{\mathcal{G}_{i}^{\prime}} \succeq \mathcal{G}_{i}^{\prime}$. We claim that any such assignment has to satisfy the condition that if $i \neq j$, then $\overline{\mathcal{G}_{i}^{\prime}} \neq \overline{\mathcal{G}_{j}^{\prime}}$. To see this, note that for any pair $\left(\mathcal{G}_{i}^{\prime}, \mathcal{G}_{j}^{\prime}\right)$, there is no principal subset $\mathcal{G}^{\prime}$ such that $\mathcal{G}^{\prime} \succeq \mathcal{G}_{i}^{\prime}$ and $\mathcal{G}^{\prime} \succeq \mathcal{G}_{j}^{\prime}$. Indeed, if it were the case, then $\operatorname{Co}\left(\mathcal{G}_{i}^{\prime}\right) \cup \mathrm{Co}\left(\mathcal{G}_{j}^{\prime}\right) \subseteq \mathrm{Co}\left(\mathcal{G}^{\prime}\right)$ and we can derive a cover of $\mathbb{A}_{G}(w)$, from (11), by replacing the two sets $\mathrm{Co}\left(\mathcal{G}_{i}^{\prime}\right)$ and $\mathrm{Co}\left(\mathcal{G}_{j}^{\prime}\right)$ with a single set $\mathrm{Co}\left(\mathcal{G}^{\prime}\right)$, which contradicts the assumption that (11) is a minimal cover of $\mathbb{A}_{G}(w)$.

Since $\overline{\mathcal{G}_{i}^{\prime}} \neq \overline{\mathcal{G}_{j}^{\prime}}$ for all $i \neq j$, we can assume, without loss of generality, that $\overline{\mathcal{G}_{i}^{\prime}}=\mathcal{G}_{i}^{*}$ for all $i=1, \ldots, l^{\prime}$. So, $\mathcal{G}_{i}^{*} \succeq \mathcal{G}_{i}^{\prime}$, and hence

$$
\mathrm{Co}\left(\mathcal{G}_{i}^{\prime}\right) \subseteq \operatorname{Co}\left(\mathcal{G}_{i}^{*}\right) .
$$

Moreover, by Lemma 8 , the equality above holds if and only if $\mathcal{G}_{i}^{\prime}=\mathcal{G}_{i}^{*}$. Since $\operatorname{Co}\left(\mathcal{G}_{1}^{*}\right), \ldots, \operatorname{Co}\left(\mathcal{G}_{l}^{*}\right)$ are pairwise disjoint; we thus conclude that

$$
\cup_{i=1}^{l^{\prime}} \operatorname{Co}\left(\mathcal{G}_{i}^{\prime}\right) \subseteq \cup_{i=1}^{l} \operatorname{Co}\left(\mathcal{G}_{i}^{*}\right) .
$$

The equality holds if and only if $\left\{\mathcal{G}_{1}^{\prime}, \ldots, \mathcal{G}_{l^{\prime}}^{\prime}\right\}=$ $\left\{\mathcal{G}_{1}^{*}, \ldots, \mathcal{G}_{l}^{*}\right\}$. In other words, we have shown that $\mathcal{S}$ is the unique minimal cover of $\mathbb{A}_{G}(w)$.

We conclude this subsection by relating $\mathbb{A}_{G}(w)$ to $\widehat{\mathbb{A}}_{G}(w)$ :

Proposition 4 Let $G=(V, E)$ be strongly connected, and $w$ be in $\operatorname{Sp}(V)$. Then, the closure of $\mathbb{A}_{G}(w)$ in $\mathbb{R}^{n \times n}$ is $\widehat{\mathbb{A}}_{G}(w)$.

Proof. If $G$ is comprised of only one vertex, then $\mathbb{A}_{G}(w)=$ $\widehat{\mathbb{A}}_{G}(w)=\{0\}$. Henceforth, we assume that the number of vertices of $G$ is greater than one. First, note that $\mathbb{A}_{G}(w)$ is contained in $\widehat{\mathbb{A}}_{G}(w)$ while $\widehat{\mathbb{A}}_{G}(w)$ is a closed set. So the closure of $\mathbb{A}_{G}(w)$ must be contained in $\widehat{\mathbb{A}}_{G}(w)$. We now show that the converse is also true, that is $\widehat{\mathbb{A}}_{G}(w)$ is contained in the closure of $\mathbb{A}_{G}(w)$. Choose a matrix $A$ in
$\widehat{\mathbb{A}}_{G}(w)-\mathbb{A}_{G}(w)$; then by Proposition 3 , we have $A=$ $\sum_{i=1}^{k} \alpha_{i} C_{i}$, with each $\alpha_{i}$ non-negative. Since for each positive real number $\epsilon>0$, the matrix $A(\epsilon):=\sum_{i=1}^{k}\left(\alpha_{i}+\epsilon\right) C_{i}$ is contained in $\mathbb{A}_{G}(w), A$ is in the closure of $\mathbb{A}_{G}(w)$. This completes the proof.

\section{Implementations of $w$-feasible Dynamics}

In this section, we assume that $G$ is a rooted digraph, and $w$ is a vector for which the set $\mathbb{A}_{G}(w)$ is nonempty. We present here a decentralized algorithm that allows the agents to find a matrix $A$ in $\mathbb{A}_{G}(w)$. In particular, we assume that each agent $x_{i}$ only knows its own weight $w_{i}$, and each agent is only able to communicate/cooperate with its neighbors, which are defined as the agents connected to $i$ with either an incoming or an outgoing edge.

\subsection{The Algorithm Generating w-Feasible Dynamics}

The implementation of the algorithm derived here relies on decentralized methods for solving the graph balancing problems. To see this, we first assume that $G=(V, E)$ is strongly connected, and $w$ is in $\operatorname{Sp}(V)$. Suppose that there is a decentralized algorithm to find a positive solution $\left\{b_{i j} \mid\right.$ $i \rightarrow j \in E\}$ of $G$-balancing. Then by Lemma 5, it suffices for each agent $x_{i}$ to set the interaction weights as $a_{i j}:=$ $b_{i j} / w_{i}$, because the resulting set $\left\{a_{i j} \mid i \rightarrow j \in E\right\}$ yields a matrix $A$ in $\mathbb{A}_{G}(w)$. So for $G$ a strongly connected digraph and $w$ a vector in $\operatorname{Sp}(V)$, the problem of finding a matrix $A \in \mathbb{A}_{G}(w)$ is reduced to the problem of finding a positive solution of $G$-balancing. This is a well studied problem, and we provide here a decentralized iterative algorithm for agents to find a positive solution of $G$-balancing

\section{Algorithm 1 ( $G$-balancing for $G$ strongly connected)}

Let $b_{i j}[l]$ be the value of $b_{i j}$ at iteration step $l \geq 0$; then, the algorithm goes as follows:

Initialization. Each agent $x_{i}$ sets $b_{i j}[0]=1$ for all $j \in V_{i}^{-}$. Iteration step. Each agent $x_{i}$ updates $b_{i j}[l]$ as follows:

$$
b_{i j}[l+1]=\frac{1}{2} b_{i j}[l]+\frac{1}{2} \sum_{k \in V_{i}^{+}} b_{k i}[l] /\left|V_{i}^{-}\right| .
$$

We refer to [27] for a proof of convergence of the algorithm.

We now consider the general case where $G$ is rooted, but not necessarily strongly connected. We assume that the vector $w$ is chosen so that $\mathbb{A}_{G}(w)$ is nonempty. For simplicity, but without loss of generality, we assume that only the first $m$ entries of $w$ are nonzero, and let $w^{\prime}$ be in $\mathbb{R}^{m}$ so that $w=\left(w^{\prime}, 0\right)$. Let $G^{\prime}=\left(V^{\prime}, E^{\prime}\right)$ be the subgraph of $G$ induced by the first $m$ vertices, and let $\mathbb{A}_{G^{\prime}}\left(w^{\prime}\right)$ be the set of relaxed $w^{\prime}$-feasible dynamics associated with $G^{\prime}$. Similarly, we partition a matrix $A \in \mathbb{A}_{G}$ into $2 \times 2$ blocks $A=\left(A_{11}, A_{12} ; A_{21}, A_{22}\right)$, with $A_{11}$ being $m \times m$. We will now describe a decentralized algorithm for agents to construct the block matrices $A_{11}, A_{12}, A_{21}$ and $A_{22}$ so that the resulting matrix $A$ is in $\mathbb{A}_{G}(w)$. The algorithm, described below, implements the construction of a specific choice of 
matrix $A \in \mathbb{A}_{G}(w)$ given in the proof of Proposition 2. Precisely, we construct the blocks $A_{i j}$ 's in a way such that the following three conditions hold:

C1. The block matrix $A_{11}$ is contained in $\mathbb{A}_{G^{\prime}}\left(w^{\prime}\right)$;

$C 2$. The block matrix $A_{12}$ is a zero matrix;

C3. The two block matrices $A_{21}$ and $A_{22}$ satisfy the condition that if $i \rightarrow j$ is an edge of $G$ with $i>m$, then $a_{i j}$, the $i j$-th entry of $A$, is 1 .

This is then achieved by the following algorithm:

Algorithm 2 (Computing an $A \in \mathbb{A}_{G}(w)$ for $G$ rooted) Initialization. Each agent $x_{i}$ informs her neighbors (both incoming and outgoing) whether her weight $w_{i}$ is zero, and reciprocally agent $x_{i}$ receives the information of weights from all of her neighbors. There are two different cases depending on whether the weight $w_{i}$ of agent $x_{i}$ is zero or not.

a). If $w_{i}=0$, then agent $x_{i}$ sets $a_{i j}[0]=1$ for all $j \in V_{i}^{-}$.

b). If $w_{i}>0$, then agent $x_{i}$ defines the sets

$$
\left\{\begin{array}{l}
V_{i}^{\prime+}:=\left\{k \in V_{i}^{+} \mid w_{k}>0\right\}, \\
V_{i}^{\prime-}:=\left\{j \in V_{i}^{-} \mid w_{j}>0\right\} .
\end{array}\right.
$$

We note that $V_{i}^{\prime+}$ (resp. $V_{i}^{\prime-}$ ) is just the set of incoming (resp. outgoing) neighbors of $i$ in the digraph $G^{\prime}$. Agent $x_{i}$ then sets

$$
a_{i j}[0]= \begin{cases}1 / w_{i} & \text { if } j \in V_{i}^{\prime-}, \\ 0 & \text { if } j \notin V_{i}^{\prime-} .\end{cases}
$$

Iteration step. We still consider two cases:

a). If $w_{i}=0$, then agent $x_{i}$ retains the value of $a_{i j}[l]$, i.e., $a_{i j}[l+1]=a_{i j}[l]=1$ for all $j \in V_{i}^{-}$.

b). If $w_{i}>0$, then agent $x_{i}$ updates $a_{i j}[l]$ as follows:

$$
a_{i j}[l+1]= \begin{cases}\frac{1}{2} a_{i j}[l]+\frac{1}{2} \frac{\sum_{k \in V_{i}^{\prime+}} w_{k} a_{k i}[l]}{w_{i}\left|V_{i}^{\prime-}\right|} & \text { if } j \in V_{i}^{\prime-}, \\ 0 & \text { if } j \notin V_{i}^{\prime-} .\end{cases}
$$

The convergence of Algorithm 2 follows from the convergence of Algorithm 1. Indeed, let $A[\cdot] \in A_{G}$ be defined such that the $i j$-th entry of $A[\cdot]$, for $i \rightarrow j \in E$, is $a_{i j}[\cdot]$. Partition the matrix $A[\cdot]$ into $2 \times 2$ blocks as

$$
A[\cdot]=\left(A_{11}[\cdot], A_{12}[\cdot] ; A_{21}[\cdot], A_{22}[\cdot]\right) \text {. }
$$

Then, from Algorithm 2, each of the three blocks $A_{12}[\cdot]$, $A_{21}[\cdot]$, and $A_{22}[\cdot]$ keeps the same value along the iterations. Moreover, $A_{12}[\cdot]$ satisfies condition $C 2$, and $A_{21}[\cdot]$ and $A_{22}[\cdot]$ satisfy condition $C 3$. For the block $A_{11}[\cdot]$, note that if $a_{i j}[\cdot]$ is replaced with $b_{i j}[\cdot] / w_{i}$ for all $i \rightarrow j \in E^{\prime}\left(w_{i} \neq 0\right.$ for $i \in V^{\prime}$ ), then we actually recover Algorithm 1 and obtain a positive solution $\left\{b_{i j} \mid i \rightarrow j \in E^{\prime}\right\}$ of $G^{\prime}$-balancing. We thus conclude from Algorithm 1 and Lemma 5 that $A_{11}[\cdot]$ converges to a matrix in $\mathbb{A}_{G^{\prime}}\left(w^{\prime}\right)$. Thus, the condition $C 1$ is also satisfied.

\subsection{Simulation study}

We present here simulation results, providing empirical evidence of the convergence of the algorithms described in the previous section. We consider three directed graphs with $n=8$ vertices: the complete graph with one edge removed, a cycle graph with an additional edge between vertices 1 and 2, and the rooted digraph depicted in Fig. 1. We removed one edge from the complete graph (resp. added one edge to the cycle graph) because for the complete graph (resp. the cycle graph), the initialization step of the algorithm already yields a $w$-feasible dynamics.

In simulations, the measure of convergence we used is $\left\|w[l]-w^{*}\right\|$ where $w^{*}$ is the (feasible) objective weight vector and $w[l]$ is the (unique) left eigenvector of $A[l]$ associated with the zero eigenvalue. The algorithm has thus converged when Error $:=\left\|w[l]-w^{*}\right\|$ is smaller than a preset tolerance.

In Fig. 2a, we show the result for the complete graph with one edge removed. Each of the four curves corresponds to an objective weight vector $w^{*}$, which has been chosen uniformly at random in the interior of the simplex. We observe that the algorithm converges monotonically at the same rate independently of the initial condition. In Fig. 2b, we performed a similar simulation for the case of the cycle graph with an added edge. The algorithm converges but as we can observe, the convergence is slower than in the previous case due to the fact that the underlying graph is sparser. Finally, in Fig. 2c, we show the result for the graph of Fig. 1. Each of the four curves corresponds to an objective weight vector $w^{*}$ obtained as follows. The bottom curve corresponds to a random weight vector with only its first 3 entries nonzero (and note that the three vertices form a relevant subset). The second curve from the bottom corresponds to a random weight vector with only its first 5 entries non-zero, and the top two curves correspond to random weight vectors with their first 7 entries non-zero. We observe that the algorithm converges in all these cases, but with convergence rates depending on the facet of the unit simplex to the interior of which the objective weight vector belongs.

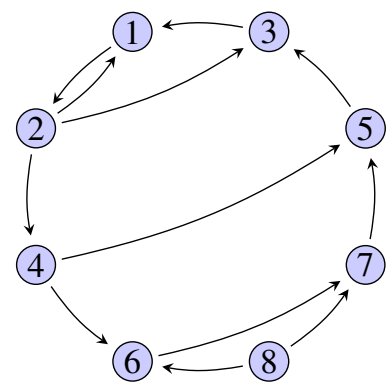

Fig. 1. A rooted graph with the root set $V_{r}=\{1,2,3,4,5,6,7\}$. Relevant subsets are $\{1\}, \ldots,\{7\},\{1,2,3\},\{1,2,3,4,5\}$, and $V_{r}$.

\section{Conclusions}

In this paper, we have addressed the following problem: given a rooted digraph $G=(V, E)$, what kind of linear 


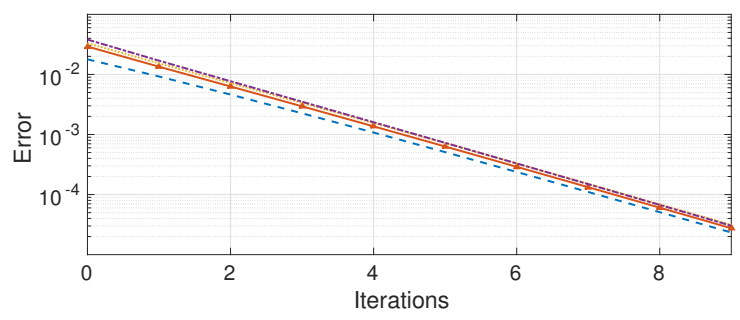

(a)

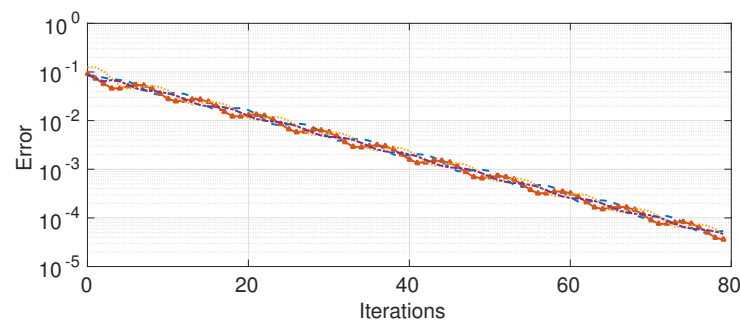

(b)

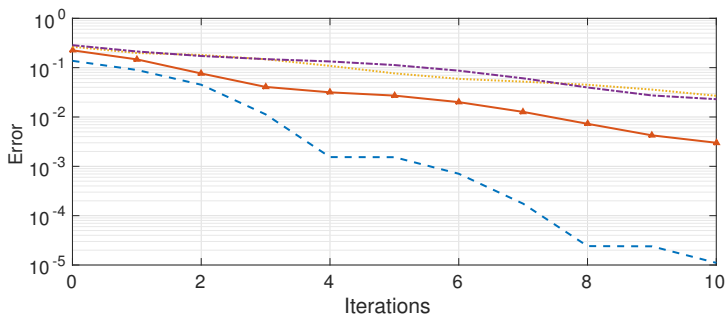

(c)

Fig. 2. Simulation results for (a) the complete graph on 8 vertices with one edge removed, (b) for the cycle graph on 8 vertices with an added edge between vertices 1 and 2 and (c) for the graph depicted in Fig. 1.

objective map $f_{w}\left(x_{1}, \ldots, x_{n}\right)=\sum_{i=1}^{n} w_{i} x_{i}$ is feasible by a choice of interaction weights $a_{i j}$ for $i \rightarrow j \in E$, where by feasible, we mean that the agents asymptotically agree on the state $f_{w}\left(x_{1}(0), \ldots, x_{n}(0)\right)$ under the dynamics $\dot{x}(t)=$ $A x(t)$ with $A=\left(a_{i j}\right)_{i j}$.

By introducing the notion of relevant subsets of vertices, we have provided a complete answer to this question in Theorem 1 for the case of continuous-time dynamics, and in Corollary 1 for the case of discrete-time dynamics. Further, by looking at cycles of $G$, and introducing the notion of principal subsets, we have characterized in Theorem 2 the set of $w$-feasible dynamics via a canonical decomposition related to the maximal elements in the collection of principal subsets. Finally, we have presented a decentralized algorithm for agents in a network to implement a selected set of interaction weights that achieves a feasible linear objective map.

Future work may focus on the case where the interaction weights $a_{i j}$ 's are allowed to be negative. The question about feasibility of a linear objective map, and the question about decentralized implementation can still be raised in this con- text for a given digraph $G$. Other open problems, such as dealing with time-varying digraphs, dealing with nonlinear objective maps, and dealing with the presence of a malicious player who attempts to increase her own weight, as in [28], are all in our future scope.

\section{Acknowledgement}

The authors acknowledge useful discussions with Dr. Ji Liu at the Coordinated Science Lab., University of Illinois at Urbana-Champaign.

\section{References}

[1] J.N. Tsitsiklis. Problems in Decentralized Decision Making and Computation. PhD thesis, Department of Electrical Engineering and Computer Science, MIT, 1984.

[2] A. Jadbabaie, J. Lin, and A.S. Morse. Coordination of groups of mobile autonomous agents using nearest neighbor rules. IEEE Transactions on Automatic Control, 48(6):988-1001, 2003.

[3] R. Olfati-Saber and R.M. Murray. Consensus problems in networks of agents with switching topology and time-delays. IEEE Transactions on Automatic Control, 49(9):1520-1533, 2004.

[4] L. Moreau. Stability of multiagent systems with time-dependent communication links. IEEE Transactions on Automatic Control, 50(2):169-182, 2005.

[5] W. Ren and R.W. Beard. Consensus seeking in multiagent systems under dynamically changing interaction topologies. IEEE Transactions on Automatic Control, 50(5):655-661, 2005.

[6] J.M. Hendrickx and J.N. Tsitsiklis. A new condition for convergence in continuous-time consensus seeking systems. In 50th IEEE Conference on Decision and Control and European Control Conference (CDC-ECC), pages 5070-5075, 2011.

[7] J. Liu, A. Nedić, and T. Başar. Internal stability of linear consensus processes. In 53rd IEEE Conference on Decision and Control (CDC), pages 922-927, 2014.

[8] M. Cao, A.S. Morse, and B.D.O. Anderson. Reaching a consensus in a dynamically changing environment: a graphical approach. SIAM Journal on Control and Optimization, 47(2):575-600, 2008.

[9] A. Kashyap, T. Başar, and R. Srikant. Quantized consensus. Automatica, 43(7):1192-1203, 2007.

[10] T. Başar, S.R. Etesami, and A. Olshevsky. Fast convergence of quantized consensus using Metropolis chains. In 53rd IEEE Conference on Decision and Control (CDC), pages 1330-1334, 2014.

[11] S.R. Etesami and T. Başar. Convergence time for unbiased quantized consensus over static and dynamic networks. IEEE Transactions on Automatic Control, 61(4), 2016. to appear.

[12] Z. Qu, C. Li, and F. Lewis. Cooperative control with distributed gain adaptation and connectivity estimation for directed networks. International Journal of Robust and Nonlinear Control, 24(3):450476, 2014.

[13] J. Liu, S. Mou, A.S. Morse, B.D.O. Anderson, and C. Yu. Deterministic gossiping. Proceedings of the IEEE, 99(9):1505-1524, 2011.

[14] M.C. De Gennaro and A. Jadbabaie. Decentralized control of connectivity for multi-agent systems. In 45th IEEE Conference on Decision and Control, pages 3628-3633, 2006.

[15] Y. Kim and M. Mesbahi. On maximizing the second smallest eigenvalue of a state-dependent graph laplacian. IEEE Transactions on Automatic Control, 51(1):116-120, 2006.

[16] C.T. Lin. Structural controllability. IEEE Transactions on Automatic Control, 19(3):201-208, 1974. 
[17] X. Chen, M.-A. Belabbas, and T. Başar. Controllability of formations over directed graphs. In 54th IEEE Conference on Decision and Control (CDC), 2015.

[18] M.-A. Belabbas. Sparse stable systems. Systems \& Control Letters, 62(10):981-987, 2013.

[19] M. Lorenzen and M.-A. Belabbas. Distributed local stabilization in formation control. In European Control Conference (ECC), pages 2914-2919. IEEE, 2014.

[20] X. Chen, M.-A. Belabbas, and T. Başar. Formations control with triangulated Laman graphs. In 54th IEEE Conference on Decision and Control (CDC), 2015.

[21] X. Chen, M.-A. Belabbas, and T. Başar. Consensus with linear objective maps. In 54th IEEE Conference on Decision and Control (CDC), 2015.

[22] L. Hooi-Tong. On a class of directed graphs-with an application to traffic-flow problems. Operations Research, 18(1):87-94, 1970.

[23] D.A. Gregory, S.J. Kirkland, and N.J. Pullman. Row-stochastic matrices with a common left fixed vector. Linear Algebra and Its Applications, 169:131-149, 1992.

[24] V. Klee and C. Witzgall. Facets and vertices of transportation polytopes. Mathematics of The Decision Sciences, 1:257-282, 1968.

[25] V.A. Yemelicher, M.M. Kovalev, M.K. Dravtsov, and G. Lawden. Polytopes, Graphs and Optimisation. Cambridge University Press, 1984.

[26] B. Gharesifard and J. Cortés. Distributed strategies for generating weight-balanced and doubly stochastic digraphs. European Journal of Control, 18(6):539-557, 2012.

[27] A. Rikos, T. Charalambous, and C.N. Hadjicostis. Distributed weight balancing over digraphs. IEEE Transactions on Control of Network Systems, 1(2):190-201, 2014.

[28] M. El Chamie and T. Başar. Optimal strategies for dynamic weight selection in consensus protocols in the presence of an adversary. In 53rd IEEE Conference on Decision and Control (CDC), pages 735-740, 2014.

\section{Appendix}

Proof of Lemma 9. In the proof, we assume, without loss of generality, that $\mathcal{G}_{i}=\left\{G_{1}, \ldots, G_{m}\right\}$ and $\mathcal{G}_{j}=\left\{G_{1}, \ldots, G_{m^{\prime}}\right\}$ with $m>m^{\prime}$. We prove Lemma 9 item by item.

1. Proof of item 1. We first show that $\mathrm{Co}\left(\mathcal{G}_{i}\right) \supseteq \operatorname{Co}\left(\mathcal{G}_{j}\right)$, then show that $\operatorname{Co}\left(\mathcal{G}_{i}\right) \neq \operatorname{Co}\left(\mathcal{G}_{j}\right)$. Since $L\left(\mathcal{G}_{i}\right)=L\left(\mathcal{G}_{j}\right)$, for each $G_{s}$ with $s>m^{\prime}$, there are coefficients $\sigma_{s t}$ 's such that $C_{s}=\sum_{t=1}^{m^{\prime}} \sigma_{s t} C_{t}$. Now, let $A=\sum_{t=1}^{m^{\prime}} \alpha_{t} C_{t} \in \operatorname{Co}\left(\mathcal{G}_{j}\right)$, with each $\alpha_{t}>0$; we show that there are positive coefficients $\beta_{s}$ 's such that $A=\sum_{s=1}^{m} \beta_{s} C_{s} \in \mathrm{Co}\left(\mathcal{G}_{i}\right)$. Choose a positive number $\epsilon$, and define

$$
\beta_{s}:= \begin{cases}\alpha_{s}-\epsilon \sum_{t=m^{\prime}+1}^{m} \sigma_{t s} & \text { if } 1 \leq s \leq m^{\prime} \\ \epsilon & \text { if } s>m^{\prime} .\end{cases}
$$

Since $\alpha_{s}>0$, we can choose $\epsilon$ sufficiently small so that $\beta_{s}>0$ for all $s=1, \ldots, m$. A direct computation then yields the following:

$$
\sum_{s=1}^{m} \beta_{s} C_{s}=\sum_{s=1}^{m^{\prime}} \alpha_{s} C_{s}+\epsilon \sum_{s=m^{\prime}+1}^{m}\left(C_{s}-\sum_{t=1}^{m^{\prime}} \sigma_{s t} C_{t}\right) .
$$

By the fact that $C_{s}=\sum_{t=1}^{m^{\prime}} \sigma_{s t} C_{t}$, the second term on the right hand side of the equation above vanishes, and hence $A=\sum_{s=1}^{m} \beta_{s} C_{s} \in \operatorname{Co}\left(\mathcal{G}_{i}\right)$.

Next, we show that there exists a matrix $A \in \operatorname{Co}\left(\mathcal{G}_{i}\right)-$ $\mathrm{Co}\left(\mathcal{G}_{j}\right)$. Consider the cycle $G_{m} \in \mathcal{G}_{i}-\mathcal{G}_{j}$ : by Proposition 3 , $\left\{\alpha C_{m} \mid \alpha>0\right\}$ is an extreme ray of $\widehat{\mathbb{A}}_{G}(w)$, and hence, there does not exist a set of non-negative coefficients $\alpha_{i}$ 's such that $C_{m}=\sum_{i=1}^{m^{\prime}} \alpha_{i} C_{i}$. This, in particular, implies that

$$
\inf \left\{\left\|C_{m}-C\right\|_{F} \mid C \in \mathrm{Co}\left[\mathcal{G}_{j}\right]\right\}>0,
$$

where $\|A\|_{F}=\operatorname{trace}\left(A^{\top} A\right)$ is the Frobenius norm of a matrix $A$. The inequality above implies that the singleton $\left\{C_{m}\right\}$ and the convex cone $\mathrm{Co}\left[\mathcal{G}_{j}\right]$ are separable via open neighborhoods. Thus, if we let $A:=C_{m}+\epsilon \sum_{i=1}^{m-1} C_{i}$, then for sufficiently small $\epsilon>0$, we have $A \in \mathrm{Co}\left(\mathcal{G}_{i}\right)-\mathrm{Co}\left[\mathcal{G}_{j}\right]$.

2. Proof of item 2. We first show that $\operatorname{Co}\left(\mathcal{G}_{i}\right) \supseteq \operatorname{Co}\left(\mathcal{G}_{j}\right)$, and then show that $\operatorname{Co}\left(\mathcal{G}_{i}\right) \neq \operatorname{Co}\left(\mathcal{G}_{j}\right)$. Let $A=\sum_{t=1}^{m^{\prime}} \alpha_{t} C_{t}$, with each $\alpha_{t}>0$; we show that there are positive coefficients $\beta_{s}$ 's such that $A=\sum_{s=1}^{m} \beta_{s} C_{s}$. Recall that $\mathcal{G}^{\prime}=$ $\mathcal{G}_{i}-\mathcal{G}_{j}$. Since $\operatorname{Co}\left(\mathcal{G}^{\prime}\right)$ intersects $L\left(\mathcal{G}_{j}\right)$, there are positive coefficients $\tilde{\beta}_{s}$ 's and coefficients $\gamma_{t}$ 's such that

$$
\sum_{s=m^{\prime}+1}^{m} \tilde{\beta}_{s} C_{s}=\sum_{t=1}^{m^{\prime}} \gamma_{t} C_{t}
$$

Now, choose a positive number $\epsilon$, and define

$$
\beta_{s}:= \begin{cases}\alpha_{s}-\epsilon \gamma_{s} & \text { if } 1 \leq s \leq m^{\prime} \\ \epsilon \tilde{\beta}_{s} & \text { if } s>m^{\prime}\end{cases}
$$

Then, for sufficiently small $\epsilon$, we have $\beta_{s}>0$ for all $s=$ $1, \ldots, m$. By the choice of $\beta_{s}$ 's, we have

$$
\sum_{s=1}^{m} \beta_{s} C_{s}=\sum_{s=1}^{m^{\prime}} \alpha_{s} C_{s}+\epsilon\left(\sum_{s=m^{\prime}+1}^{m} \tilde{\beta}_{s} C_{s}-\sum_{s=1}^{m^{\prime}} \gamma_{s} C_{s}\right) .
$$

From (12), the second term on the right hand side vanishes, and hence $A=\sum_{s=1}^{m} \beta_{s} C_{s} \in \mathrm{Co}\left(\mathcal{G}_{i}\right)$.

Next, we show that there exists a matrix $A \in \mathrm{Co}\left(\mathcal{G}_{i}\right)-$ $\mathrm{Co}\left(\mathcal{G}_{j}\right)$. This holds because $\operatorname{Co}\left(\mathcal{G}_{i}\right)$ is an open set in $L\left(\mathcal{G}_{i}\right)$ and, by assumption, $\operatorname{dim} L\left(\mathcal{G}_{i}\right)>\operatorname{dim} L\left(\mathcal{G}_{j}\right)$. So, there exists a matrix $A \in \mathrm{Co}\left(\mathcal{G}_{i}\right)-L\left(\mathcal{G}_{j}\right)$.

Proof of item 3. We prove this case by contradiction. Suppose that $A=\sum_{t=1}^{m^{\prime}} \alpha_{t} C_{t}=\sum_{s=1}^{m} \beta_{s} C_{s}$, with $\alpha_{t}$ 's and $\beta_{s}$ 's positive coefficients; then,

$$
\sum_{s=m^{\prime}+1}^{m} \beta_{s} C_{s}=\sum_{s=1}^{m^{\prime}}\left(\alpha_{t}-\beta_{t}\right) C_{t} \in \operatorname{Co}\left(\mathcal{G}^{\prime}\right) \cap L\left(\mathcal{G}_{j}\right),
$$

which is a contradiction. This completes the proof. 